\title{
Oral Squamous Cell Carcinoma: Epidemiology, Clinical Presentation and Treatment
}

\author{
Liviu Feller, Johan Lemmer
}

Department of Periodontology and Oral Medicine, University of Limpopo, Medunsa Campus, Pretoria, South Africa. Email: lfeller@ul.ac.za

Received June $15^{\text {th }}$, 2012; revised July $20^{\text {th }}$, 2012; accepted July $31^{\text {st }}, 2012$

\begin{abstract}
Squamous cell carcinoma accounts for $90 \%$ of all oral cancers. It may affect any anatomical site in the mouth, but most commonly the tongue and the floor of the mouth. It usually arises from a pre-existing potentially malignant lesion, and occasionally de novo; but in either case from within a field of precancerized epithelium. The use of tobacco and betel quid, heavy drinking of alcoholic beverages and a diet low in fresh fruits and vegetables are well known risk factors for oral squamous cell carcinoma. Important risk factors related to the carcinoma itself that are associated with a poor prognosis include large size of the tumour at the time of diagnosis, the presence of metastases in regional lymphnodes, and a deep invasive front of the tumour. Squamous cell carcinoma is managed by surgery, radiation, and chemotherapy singularly or in combination; but regardless of the treatment modality, the five-year survival rate is poor at about $50 \%$. This can be attributed to the fact that about two-thirds of persons with oral squamous cell carcinoma already have a large lesion at the time of diagnosis.
\end{abstract}

Keywords: Oral Squamous Cell Carcinoma; Epidemiology; Clinical Course; Field of Precancerization

\section{Introduction}

Oral cancer is the sixth most common cancer worldwide [1]. More than $90 \%$ of all oral cancers are squamous cell carcinoma (SCC) [2,3]. The most important risk factors for oral SCC are use of tobacco or betel quid and the regular drinking of alcoholic beverages. However, infection with high-risk human papillomavirus (HPV) genotypes, and a diet low in fresh fruits and vegetables have also recently been implicated in the aetiopathogenesis of oral SCC $[1,4]$. The highest incidence and prevalence of oral SCC is found in the Indian subcontinent where the risk of developing oral SCC is increased by the very prevalent habits of chewing tobacco, betel quid and areca-nut [2]. The mutagenic effects of tobacco, alcohol, betel quid or areca-nut are dependent upon dose, upon frequency and upon duration of use, and are accelerated and exaggerated by the concurrent use of two or more of these agents [4].

However, as not all persons who practice these highrisk habits will develop oral SCC, and as oral SCC may be idiopathic, there must be person-specific genetic characteristics and environmental factors which may either afford protection against the development of oral SCC, or may predispose to or even promote the development of oral SCC.

In the last 30 years, the 5-year survival rate of patients with oral SCC has not improved despite advances in diagnostic techniques and improvements in treatment modalities. Indeed, the incidence and prevalence of oral SCC are increasing, particularly in younger persons $[5,6]$.

The aim of this article is to review the epidemiology, clinical features, and prognosis of oral SCC.

\section{Epidemiology of Oral SCC}

Oral SCC more frequently affects men than women (M:F $=1.5: 1)$ most probably because more men than women indulge in high-risk habits. The probability of developing oral SCC increases with the period of exposure to risk factors, and increasing age adds the further dimension of age-related mutagenic and epigenetic changes. In the USA the median age of diagnosis of oral SCC is 62 years. However, the incidence of oral SCC in persons under the age of 45 is increasing [7]. The reason for this is obscure.

A number of conditions have been associated with an elevated risk of developing oral SCC including Li Fraumeni syndrome, Plummer-Vinson syndrome, Fanconi anemia, chemotherapy induced immunosuppression of organ transplantation, dyskeratosis congenita, xeroderma pigmentosum and discoid lupus erythematosus [8].

In Western countries oral SCC affects the tongue in $20 \%-40 \%$ of cases and the floor of the mouth in $15 \%$ $20 \%$ of the cases, and together these sites account for 
about $50 \%$ of all cases of oral SCC [3,9]. The gingivae, palate, retromolar area and the buccal and labial mucosa are oral sites less frequently affected [7].

The ventral surface of the tongue and the floor of the mouth are the sites most commonly affected by SCC because they are lined by thin non-keratinised epithelium. Not only do carcinogens readily penetrate this thin epithelium to reach the progenitor cell compartment, butcarcinogens, particularly tobacco products and alcohol in solution, constantly accumulate in the floor of the mouth and bathe the tissues of the floor of the mouth and the ventrum of tongue [5].

The mean 5-year survival rate of persons with oral SCC is about $50 \%$ with no gender difference; but black persons have a lower five year survival rate than persons of other races $[5,7,10,11]$. Other socio-demographic factors such as age, potentially carcinogenic habits (using alcohol, tobacco, betel quid) or socio-economic status are not consistently related to survival rates [9].

The stage of advancement of oral SCC at the time of diagnosis is the most important prognostic factor [8]. Oral SCC is most frequently diagnosed late in the course of the disease because affected persons fail to seek professional advice timeously, either because they do not understand the significance of early signs and symptoms, or because they are ignorant of the health implications [7].

\section{Clinical Features and Course of Oral SCC}

Oral SCC may take various clinical forms. It may resemble a leukoplakia, a verrucous leukoplakia, an erythroleukoplakia, or an erythroplakia, any of which may eventually develop into a necrotic looking ulcer with irregular, raised indurated borders, or into a broad based exophytic mass with a surface texture which may be verrucous, pebbled or relatively smooth. When traumatized, oral SCC bleeds readily and often becomes superficially secondarily infected. Oral SCC is usually painless unless it issecondarily infected. Large lesions may interfere with normal speech, mastication or swallowing $[3,5,8]$.

The course of oral SCC is unpredictable, but the TNM stage (T-tumour size, N-nodal metastasis, M-distant metastasis) of the primary tumour correlates well with the survival rate [8]. The prognosis is best when the primary tumour is small and there is no evidence of regional lymphnode involvement or distant metastasis. In fact, the 5year survival rate of persons with early-stage oral SCC according to the TNM staging system may reach $80 \%$ $90 \%$ [3], whereas the five-year survival rate for advanced-stage oral SCC is about $40 \%$ [12].

About two-thirds of oral SCC are already of substantial size, and will have clinically detectable metastases to cervical lymphnodes at the time of diagnosis [5,12-14]. The affected lymphnodes are firm and non-tender to pal- pation, and if extracapsular spread into the surrounding connective tissue has occurred, they will be fixed and matted [5]. Importantly, in about $20 \%-40 \%$ of cases with no clinical or imaging evidence of metastatic spread to lymphnodes at the time of diagnosis of oral SCC, histopathological examination of the regional lymphnodes will show metastatic growth [14]. Furthermore, in one study, 21\% of cases of SCC of the head and neck in which the regional lymphnodes appeared to be free of metastatic growth when examined microscopically, in fact, molecular analysis proved them to harbour cancerous cells [15].

The presence of extracapsular lymphnode spread is associated with a high-rate of local and regional recurrence, distant metastasis and mortality [14]. About $8 \%$ of patients with oral SCC will have distant metastases at the time of diagnosis [14], most frequently to the lungs [5].

Important factors at the time of diagnosis of oral SCCdetermining survival are the presence of regional lymphnode metastases, the size (surface dimension) and depth (extent of local infiltration) of the carcinoma, the oral anatomical site affected and the histopathological grade of the carcinoma. After treatment, factors correlating with survival will be whether or not the margins of the resected carcinoma were free of invading carcinomatous cells, because this will determine whether or not there will be local recurrence; and whether or not a second tumour will develop in the same or in a contiguous epithelialized precancerized field [8,9,16-18].

Squamous cell carcinoma of the lip, hard palate and maxillary gingiva infrequently metastasize to regional lymphnodes, usually run a relative indolent course and have a relatively favourable prognosis, while SCC of the tongue, of the floor of the mouth and of the mandibular gingivaoften metastasize to regional lymphnodes and are more aggressive with a less favourable prognosis. In general, SCCs of the posterior part of the oral cavity are much more likely to metastasize to regional lymphnodes than are comparable SCCs of the anterior part of the oral cavity [1].

Small well-differentiated, low-grade oral SCCs usually metastasize to regional lymphnodes only after invading connective tissue, muscle or bone. On the other hand, poorly-differentiated, high-grade oral SCCs are biologically more aggressive and tend to metastasize to regional lymphnodes early in the course of the disease [1].

Although the grade of histological differentiation of oral SCC reflects the aggressive capacity of the tumour, it appears that as an independent factor, it does not significantly influence the prognosis $[1,19,20]$. On the other hand, the depth of the infiltration of the tumour as determined histopathologically correlates significantly with the prognosis. Oral SCCs that have infiltrated more than $5 \mathrm{~mm}$ into the underlying tissues, are more likely to me- 
tastasize to lymphnodes with a poorer prognosis $[1,14]$.

The risk of local recurrence is greatest when there are cancerous cells present in the surgical margins, but there is an increased risk of recurrence when the carcinomafree margins are narrower than $5 \mathrm{~mm}$ or when there is still dysplastic though not frankly malignant epithelium at the margins. Regardless of the width of the carcinomafree margins, the risk of local recurrence is related to the size and to the depth of infiltration of the primary carcinoma [16,21].

Resection margins apparently free of malignant cells as determined by histopathological examination have been shown by molecular analysis to harbour transformed keratinocytes with a malignant profile [15]. Therefore, despite apparently successful treatment, persons who have had oral SCC are at heightened risk of developing recurrence at the same site, from cancerous keratinocytes left behind at surgery. A carcinoma that subsequently develops within the field of precancerized epithelium from which the primary carcinoma had arisen is technically a new carcinoma although it may be immediately contiguous to the site of the primary carcinoma $[17,18$, 22].

The risk of developing multiple oral SCCs within a cytogenetically altered precancerized field is higher in young persons $[19,23]$, and in persons who continue to use tobacco, alcohol and betel quid after successful treatment of the primary carcinoma. It has been reported that about $30 \%$ of males and $20 \%$ of females who had a primary head and neck SCC will develop a second field carcinoma within 20 years of the diagnosis of the primary carcinoma [7]. In case of oral SCC, it is estimated that $50 \%$ of second field carcinomata will affect the mouth and the oropharynx and $50 \%$ the larynx, oesophagus or lungs [24]. It appears that there are no significant differences between SCCs of particular oral mucosal sites and the risk of developing a second field carcinoma $[25,26]$. The survival rate after the appearance of a second field carcinoma is low $[23,27]$.

Oral SCC can arise from pre-existing potentially malignant disorders including oral leukoplakia, erythroplakia, submucous fibrosis and lichenoid dysplastic lesions, or can arise de novo $[8,28]$. There is a debate in the literature with regard to the malignant potential of oral lichen planus, in particular the erosive form. While some researchers found an association between oral lichen planus and development of oral SCC, others did not $[5,28]$. The view of authors of this article is that oral lichen planus does not pose an increased risk of oral SCC.

It has been suggested that oral SCC evolving from leukoplakic lesions have a better prognosis than those emerging de novo, but a recent study has shown that the prognosis is not significantly different in these two groups of oral SCC [29].

\subsection{Ethnicity and Socio-Economic Status as They Relate to Oral SCC}

There is a marked variation with regard to the incidence of and mortality from oral SCC between different countries, between different geographic locations and between ethnic/racial groups. This may be attributed to exposure to different environmental factors and to ethnic-specific high-risk habits [10].

Oral SCC is more prevalent in developing than developed countries $[7,10]$. In Israel, oral SCC is more prevalent among Ashkenazi Jews than among Sephardic-Jews probably because of their different geographic origins; [30] and in England it is more prevalent among Indian people born in the Indian subcontinent and migrated to England than among Indians born in England or among white English people [31].

In the United States, the average 5-year survival rate for black people is lower than for white people with oral SCC; $[10,11]$ and in general, oral SCC is at a significantly more advanced stage in black people than in white people at the time of diagnosis [11].

The racial disparity with regard to the stage of oral SCC, and with regard to the outcome of treatment is brought about by a complex interaction of factors. It is possible that pathobiologically oral SCC is more aggressive in blacks than in whites, or that for cultural, educational and socioeconomic reasons blacks delay longer before seeking medical advice than do whites. Thus while socioeconomic status, educational level, cultural influences and limited access to health care services do not play any direct role in the development of oral SCC, they do indirectly influence the higher morbidity and mortality from oral SCC in persons from disadvantaged backgrounds $[10,11]$.

\subsection{Prevention and Control of Oral SCC}

The overall aim of cancer prevention is to reduce the incidence of the disease; and of cancer control is to detect the disease in its initial stages and to promptly institute effective and efficient treatment [32].

Measures directed at the public to reduce the incidence of oral SCC and to alert those at risk to the benefits of early detection should include education about the riskfactors associated with the disease, about the early signs and symptoms of the disease, and about the hazards of delaying seeking professional advice. Professional measures should include the making available of immediate effective and efficient medical treatment, and of screening programmes for high-risk populations with a view of identifying potentially malignant oral disorders, or early SCC [7].

This is of paramount importance because in general, abstinence from the use of tobacco and betel quid, and 
moderation in the consumption of alcoholic beverages, together with an increase in fresh fruits and vegetables in the diet, may reduce the incidence of oral SCC by almost $80 \%$ [7].

Many healthcare practitioners do not routinely perform oral soft tissue examinations for those patients who are known to practice habits associated with increased risk of oral cancer. However, in order to increase the rate of early diagnosis of oral SCC, healthcare practitioners should make a point, whenever possible, of examining the mouth as part of a general examination. If any suspicious lesions of the oral soft tissues are detected the patient should be referred to an appropriately qualified practitioner for further investigation [5,11].

There can be little doubt that careful annual examination of the mouth in all persons above the age of 40 years will result in a significant improvement in the rate of early detection of oral cancer with all the therapeutic advantages [5]. A very obvious shortcoming of such an idealised plan is that a great proportion, if not the majority of those at risk of oral SCC do not attend annually for any healthcare.

\subsection{Treatment}

The treatment of oral SCC generally requires the services of a multidisciplinary team $[1,33]$, the primary aim of treatment always being to eradicate the cancer, to prevent recurrence, and insofar as is possible to restore the form and function of the affected parts. The selection of a specific treatment modality is dictated by the nature of the carcinoma and by the general condition of the patient. Salient factors related to the carcinoma include the specific site affected, the clinical size, the extent of local invasion, histopathological features, regional lymphnode involvement and distant metastasis. Patient factors include age, general health status, a history of previously treated oral SCC and high-risk habits [1].

A variety of modalities are available for the treatment of oral SCC. These include excision/resection, radio-therapy, systemic cytotoxic chemotherapy and blocking of epithelial growth factor receptor (EGF-R), or a combination of these, either concurrently or in an orderly sequence $[6,13]$.

Surgery is the preferred first line treatment of small, accessible oral SCCs. However, advanced-stage oral SCC is usually treated by a combined treatment program of surgery, chemotherapy, and radiotherapy [1,34]. In cases of recurrent oral SCC, EGF-R inhibitor coupled with chemoradiotherapy, is the first line of treatment [33].

Surgical resection of oral carcinoma with tumour free margins of less than $5 \mathrm{~mm}$ may be followed by local recurrence and possibly by distant metastasis, and usually necessitates the administration of post-surgery chemoradiotherapy. The importance of the presence of dysplas- tic epithelium in post-resection carcinoma-free margins is of debatable importance, but it is not usually considered to be a strong indication for further treatment [34].

Twenty to thirty percent of cases of resection of oral SCC with adequate,wider than $5 \mathrm{~mm}$, tumour-free margins as evidenced on histopathological examination will develop local or contiguous regional "recurrence" $[24,25$, 34,35]. There are two possible explanations for this highrate of recurrence. Firstly, some carcinomatous keratinocytes may have remained in the margins of the surgical wound, but because there were so few, they were not detected by histopathological examination; secondly, the large field of precancerized epithelium comprising precancerous keratinocytes at different stages of transformation from which the primary carcinoma developed, was not removed at the surgical procedure. Epithelium from a field of precancerization may appear normal microscopically, or it may be dysplastic. It may also appear normal microscopically, but nevertheless may harbour keratinocytes with cytogenetic alterations including loss of heterozygosity and p53 mutations [24,36], or epigenetic changes in methylations of certain promoters of tumoursuppressor genes and DNA repair genes [37]. Following acquisition of additional genetic alterations, either keratinocytes in the dysplastic epithelium or the genetically transformed keratinocytes may become cancerous giving rise to a new field carcinoma close to where the primary carcinoma had been excised [24,34], creating an impresssion of recurrence.

Thus, the reappearance of SCC in the immediate or general vicinity of the primary oral SCC, may be a recurrence if the two carcinomata exhibit identical genetic profiles; may be a new field carcinoma from a subclone of cells within the field if the genetic profiles of the two cancers are similar, but not identical; or may be another primary carcinoma from a different clone within the same field of precancerization if the genetic profile of the two tumours are dissimilar [24].

It would be greatly advantageous if it were possible to treat a field of precancerized oral epithelium. However, as markers which predict with any degree of certainty progression of precancerized epithelium to SCC have not yet been identified, and as only $30 \%$ of patients with primary oral SCC will develop a second field tumour, any type of treatment of a precancerized field is likely to be harmful to those $70 \%$ of patients, who were not going to develop "local recurrence".

Although a precancerized field could be identified by molecular techniques or occasionally histologically, the problem is where to take tissue samples since molecularly precancerized fields are not clinically identifiable.

\section{Summary}

Oral SCC arises from within a field of precancerized 
epithelium either from a pre-existing potentially malignnant lesion, or de novo. The use of tobacco and betel quid, heavy drinking of alcoholic beverages and a diet low in fresh fruits and vegetables are the major risk factors for oral SCC. The 5-year survival rate is poor at about 50\%, mainly because about two-thirds of persons with oral SCC already have large lesions at the time of diagnosis.

\section{REFERENCES}

[1] J. P. Shah and Z. Gil, "Current Concepts in Management of Oral Cancer-Surgery,” Oral Oncology, Vol. 45, No. 4, 2009, pp. 394-401. doi:10.1016/j.oraloncology.2008.05.017

[2] E. Attar, S. Dey, A. Hablas, I. A. Seifeldin, M. Ramadan, L. S. Rozek and A. S. Soliman, "Head and Neck Cancer in a Developing Country: A Population-Based Perspective Across 8 Years," Oral Oncology, Vol. 46, No. 8, 2010, pp. 591-596. doi:10.1016/j.oraloncology.2010.05.002

[3] J. Bagan, G. Sarrion and Y. Jimenez, "Oral Cancer: Clinical Features,” Oral Oncology, Vol. 46, No. 6, 2010, pp. 414-417. doi:10.1016/j.oraloncology.2010.03.009

[4] S. Petti, "Lifestyle Risk Factors for Oral Cancer," Oral Oncology, Vol. 45, No. 4, 2009, pp. 340-350. doi:10.1016/j.oraloncology.2008.05.018

[5] B. W. Neville and T. A. Day, "Oral Cancer and Precancerous Lesions,” CA: A Cancer Journal for Clinicians, Vol. 52, No. 4, 2002, pp. 195-215. doi:10.3322/canjclin.52.4.195

[6] A. D. Rapidis, P. Gullane, J. D. Langdon, J. L. Lefebvre, C. Scully and J. P. Shah, "Major Advances in the Knowledge and Understanding of the Epidemiology, Aetiopathogenesis, Diagnosis, Management and Prognosis of Oral Cancer,” Oral Oncology, Vol. 45, No. 4, 2009, pp. 299-300. doi:10.1016/j.oraloncology.2009.04.001

[7] S. Warnakulasuriya, "Global Epidemiology of Oral and Oropharyngeal Cancer,” Oral Oncology, Vol. 45, No. 4, 2009, pp. 309-316. doi:10.1016/j.oraloncology.2008.06.002

[8] C. Scully and J. Bagan, "Oral Squamous Cell Carcinoma Overview,” Oral Oncology, Vol. 45, No. 4, 2009, pp. 301308. doi:10.1016/j.oraloncology.2009.01.004

[9] I. O. Bello, Y. Soini and T. Salo, "Prognostic Evaluation of Oral Tongue Cancer: Means, Markers and Perspectives (I),” Oral Oncology, Vol. 46, No. 9, 2010, pp. 630-635. doi:10.1016/j.oraloncology.2010.06.006

[10] C. Scully and R. Bedi, "Ethnicity and Oral Cancer," TheLancet Oncology, Vol. 1, No. 1, 2000, pp. 37-42. doi:10.1016/S1470-2045(00)00008-5

[11] C. H. Shiboski, B. L. Schmidt and R. C. Jordan, "Racial Disparity in Stage at Diagnosis and Survival among Adults with Oral Cancer in the US," Community Dentistry and Oral Epidemiology, Vol. 35, No. 3, 2007, pp. 233-240. doi:10.1111/j.0301-5661.2007.00334.x

[12] D. Brandizzi, M. Gandolfo, M. L. Velazco, R. L. Cabrini and H. E. Lanfranchi, "Clinical Features and Evolution of oral Cancer: A Study of 274 Cases in Buenos Aires, Argentina," Medicina Oral, Patologia Oral, Cirugia Bucal, Vol. 13, No. 9, 2008, pp. 544-548.

[13] R. Mazeron, Y. Tao, A. Lusinchi and J. Bourhis, "Current Concepts of Management in Radiotherapy for Head and Neck Squamous-Cell Cancer,” Oral Oncology, Vol. 45, No. 4, 2009, pp. 402-408. doi:10.1016/j.oraloncology.2009.01.010

[14] J. Massano, F. S. Regateiro, G. Januario and A. Ferreira, "Oral Squamous Cell Carcinoma: Review of Prognostic and Predictive Factors," Oral Surgery, Oral Medicine, Oral Pathology, Oral Radiology and Endodontics, Vol. 102, No. 1, 2006, pp. 67-76.

doi:10.1016/j.tripleo.2005.07.038

[15] J. A. Brennan, L. Mao, R. H. Hruban, J. O. Boyle, Y. J. Eby, W. M. Koch, S. N. Goodman and D. Sidransky, "Molecular Assessment of Histopathological Staging in Squamous-Cell Carcinoma of the Head and Neck," New England Journal of Medicine, Vol. 332, No. 7, 1995, pp. 429-435. doi:10.1056/NEJM199502163320704

[16] S. R. Larsen, J. Johansen, J. A. Sorensen and A. Krogdahl, "The Prognostic Significance of Histological Features in Oral Squamous Cell Carcinoma," Journal of Oral Pathology and Medicine, Vol. 38, No. 8, 2009, pp. 657-662. doi:10.1111/j.1600-0714.2009.00797.x

[17] M. P. Tabor, R. H. Brakenhoff, H. J. Ruijter-Schippers, J. E. Van Der Wal, G. B. Snow, C. R. Leemans and B. J. Braakhuis, "Multiple Head and Neck Tumors Frequently Originate from a Single Preneoplastic Lesion,” American Journal of Pathology, Vol. 161, No. 3, 2002, pp. 10511060. doi:10.1016/S0002-9440(10)64266-6

[18] V. M. van Houten, C. R. Leemans, J. A. Kummer, J. Dijkstra, D. J. Kuik, M. W. van den Brekel, G. B. Snow and R. H. Brakenhoff, "Molecular Diagnosis of Surgical Margins and Local Recurrence in Head and Neck Cancer Patients: A Prospective Study," Clinical Cancer Research, Vol. 10, No. 11, 2004, pp. 3614-3620. doi:10.1158/1078-0432.CCR-03-0631

[19] R. E. Friedrich, "Primary and Second Primary Cancer in 649 Patients with Malignancies of the Maxillofacial Region,” Anticancer Research, Vol. 27, No. 4A, 2007, pp. 1805-1818.

[20] M. Weijers, G. B. Snow, P. D. Bezemer and I. van der Waal, "Malignancy Grading Is No Better than Conventional Histopathological Grading in Small Squamous Cell Carcinoma of Tongue and Floor of Mouth: Retrospective Study in 128 Patients," Journal of Oral Pathology and Medicine, Vol. 38, No. 4, 2009, pp. 343-347. doi:10.1111/j.1600-0714.2009.00751.x

[21] H. Kurita, Y. Nakanishi, R. Nishizawa, T. Xiao, T. Kamata, T. Koike and H. Kobayashi, "Impact of Different Surgical Margin Conditions on Local Recurrence of Oral Squamous Cell Carcinoma," Oral Oncology, Vol. 46, No. 11, 2010, pp. 814-817. doi:10.1016/j.oraloncology.2010.08.014

[22] L. Feller and J. Lemmer, "Field Cancerization and Oral Leukoplakia,” In: G. D. Dakubo, Ed., Field Cancerization: Basic Science and Clinical Applications, Nova Science Publishers, Ontario, 2011, pp. 95-111. 
[23] E. Rennemo, U. Zatterstrom and M. Boysen, "Impact of Second Primary Tumors on Survival in Head and Neck Cancer: An Analysis of 2063 Cases,” Laryngoscope, Vol. 118, No. 8, 2008, pp. 1350-1356. doi:10.1097/MLG.0b013e318172ef9a

[24] B. J. Braakhuis, M. P. Tabor, C. R. Leemans, I. van der Waal, G. B. Snow and R. H. Brakenhoff, "Second Primary Tumors and Field Cancerization in Oral and Oropharyngeal Cancer: Molecular Techniques Provide New Insights and Definitions,” Head and Neck, Vol. 24, No. 2, 2002, pp. 198-206. doi:10.1002/hed.10042

[25] N. de Vries, I. Van der Waal and G. B. Snow, "Multiple Primary Tumours in Oral Cancer," International Journal of Oral and Maxillofacial Surgery, Vol. 15, No. 1, 1986, pp. 85-87. doi:10.1016/S0300-9785(86)80015-1

[26] I. van der Waal and R. de Bree, "Second Primary Tumours in Oral Cancer,” Oral Oncology, Vol. 46, No. 6, 2010, pp. 426-428. doi:10.1016/j.oraloncology.2010.02.018

[27] I. J. Dhooge, M. De Vos and P. B. Van Cauwenberge, "Multiple Primary Malignant Tumors in Patients with Head and Neck Cancer: Results of a Prospective Study and Future Perspectives,” Laryngoscope, Vol. 108, No. 2, 1998, pp. 250-256. doi:10.1097/00005537-199802000-00017

[28] I. van der Waal, "Potentially Malignant Disorders of the Oral and Oropharyngeal Mucosa; terminology, Classification and Present Concepts of Management," Oral Oncology, Vol. 45, No. 4-5, 2009, pp. 317-323. doi:10.1016/j.oraloncology.2008.05.016

[29] M. Weijers, I. Ten Hove, R. H. Allard, D. P. Bezemer and I. van der Waal, "Patients with Oral Cancer Developing from Pre-Existing Oral Leukoplakia: Do They Do Better than Those with De Novo Oral Cancer?”Journal of Oral Pathology and Medicine, Vol. 37, No. 3, 2008, pp. 134136. doi:10.1111/j.1600-0714.2007.00601.X

[30] M. Gorsky, M. M. Littner, Y. Sukman and A. Begleiter, "The Prevalence of Oral Cancer in Relation to the Ethnic
Origin of Israeli Jews,” Oral Surgery, Oral Medicine, Oral Pathology, Vol. 78, No. 3, 1994, pp. 408-411. doi:10.1016/0030-4220(94)90077-9

[31] A. J. Swerdlow, M. G. Marmot, A. E. Grulich and J. Head, "Cancer Mortality in Indian and British Ethnic Immigrants from the Indian Subcontinent to England and Wales,” British Journal of Cancer, Vol. 72, No. 5, 1995, pp. 1312-1319. doi:10.1038/bjc.1995.507

[32] P. E. Petersen, "Oral Cancer Prevention and ControlThe Approach of the World Health Organization," Oral Oncology, Vol. 45, No. 4, 2009, pp. 454-460. doi:10.1016/j.oraloncology.2008.05.023

[33] J. H. Lorch, M. R. Posner, L. J. Wirth and R. I. Haddad, "Seeking Alternative Biological Therapies: The Future of Targeted Molecular Treatment,” Oral Oncology, Vol. 45, No. 4, 2009, pp. 447-453. doi:10.1016/j.oraloncology.2008.08.009

[34] B. J. Braakhuis, E. Bloemena, C. R. Leemans and R. H. Brakenhoff, "Molecular Analysis of Surgical Margins in Head and Neck Cancer: More than a Marginal Issue," Oral Oncology, Vol. 46, No. 7, 2010, pp. 485-491. doi:10.1016/j.oraloncology.2010.01.019

[35] F. Levi, V. C. Te, L. Randimbison, M. Maspoli and C. La Vecchia, "Second Primary Oral and Pharyngeal Cancers in Subjects Diagnosed with Oral and Pharyngeal Cancer," International Journal of Cancer, Vol. 119, No. 11, 2006, pp. 2702-2704. doi:10.1002/ijc.22183

[36] O. Gallo and S. Bianchi, "P53 Expression: A Potential Biomarker for Risk of Multiple Primary Malignancies in the Upper Aerodigestive Tract," European Journal of Cancer Part B, Oral Oncology, Vol. 31, No. 1, 1995, pp. 53-57. doi:10.1016/0964-1955(94)00031-X

[37] D. Goldenberg, S. Harden, B. G. Masayesva, P. Ha, N. Benoit, W. H. Westra, W. M. Koch, D. Sidransky and J. A. Califano, "Intraoperative Molecular Margin Analysis in Head and Neck Cancer," Archives of Otolaryngology_Head and Neck Surgery, Vol. 130, No. 1, 2004, pp. 39-44. doi:10.1001/archotol.130.1.39 